
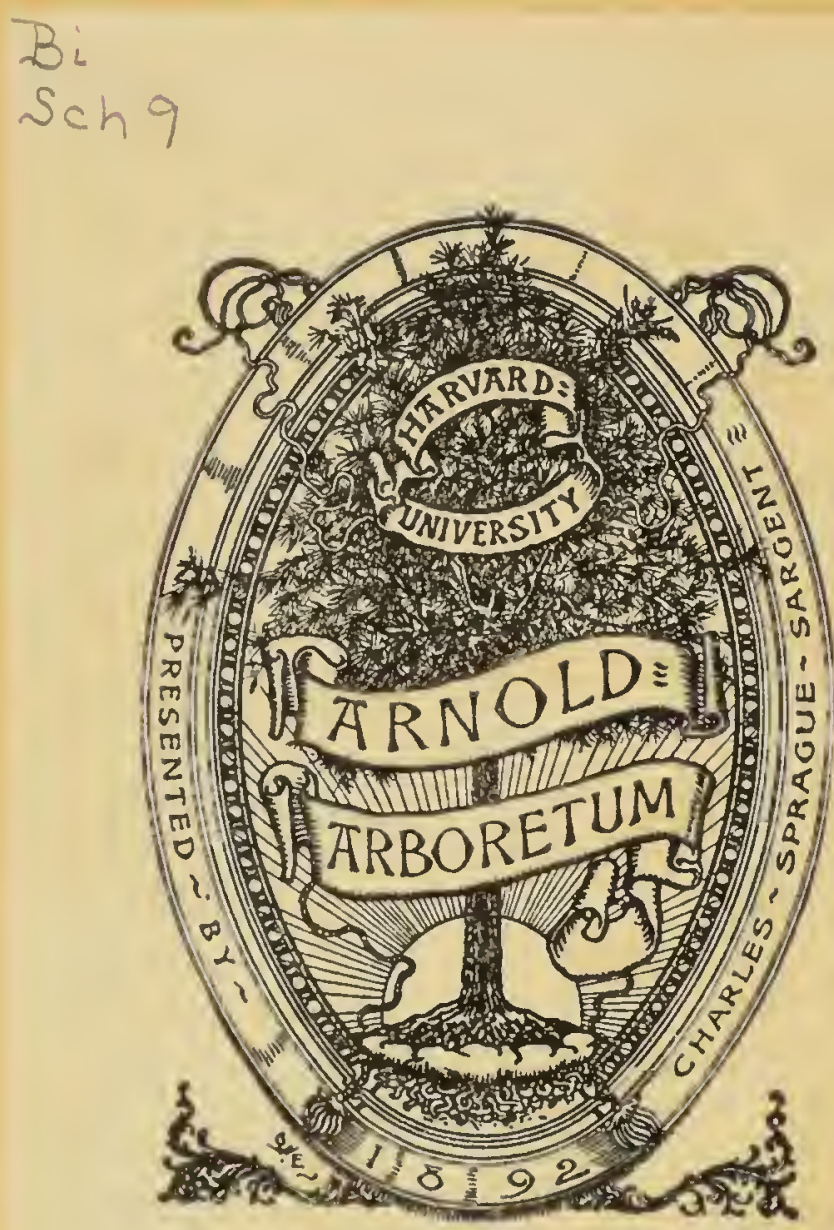




\title{
T OPOGRAPHIAE
}

\section{BOTANICAE ET ENTOM̄ŌOLOGICAE}

\author{
L I P S I E N S I S
}

S P E C,I IM E N P P I IM V IM

\section{Q $\quad$ Y $\quad 0 \quad$ D}

\section{AMPLISSIMI PHILOSOPHORVM ORDINIS}

\author{
A V C T O R I T A T E
}

DIE XIII. A PRIIIANNI M DCCXCIX

D I F E N D E T

\section{CIIRISTIANVS FRIDERICVS SCHWAEGRICHEN}

PII L. D. ET A. A. L. L. IIAG. MT DIC.BACC.SOCIETATIS

IINASAN. LIPESINSIS SODALIS

$I$ I $P$ S I I E E

$\begin{array}{lllll}S & O & C & I & O\end{array}$

IOANNO CHRISTIANO AVGVSTO CLARO MIED. DACC. SOCIET. LINNAEAN. LIPBIENSIS SODALI

COBVROC-ERAN CO

\section{I P S I A E}

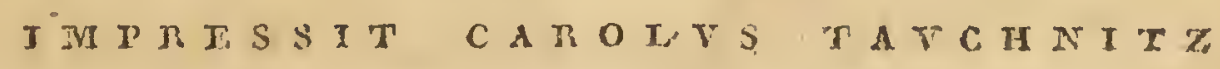


$\therefore$ initions3o in:

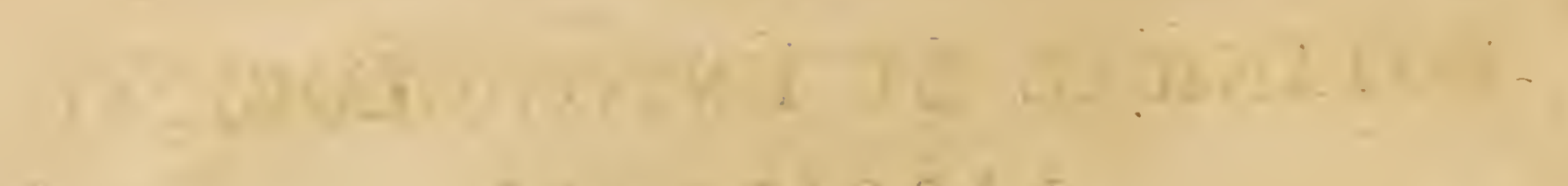

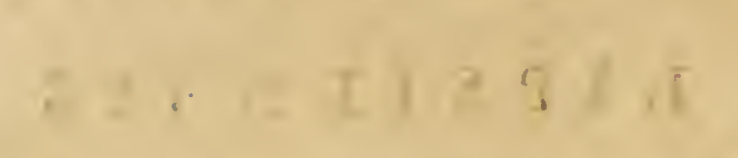

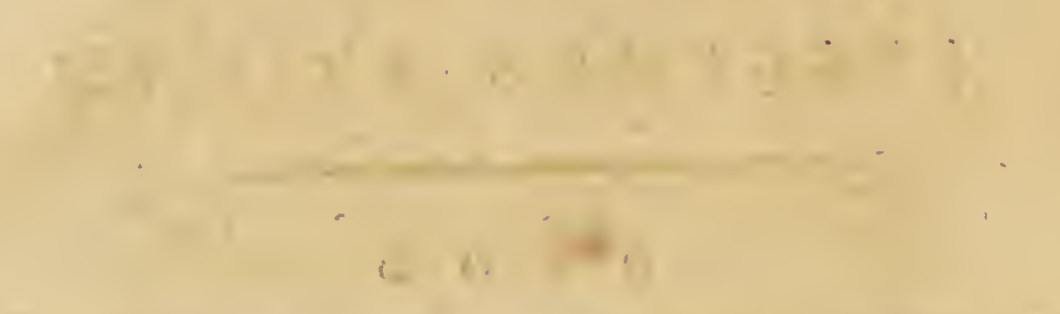

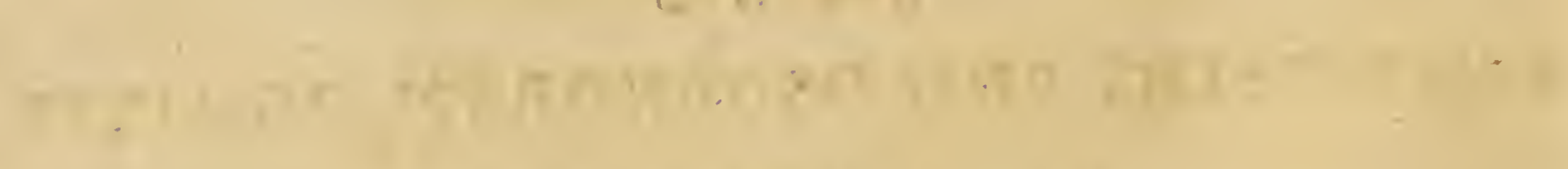

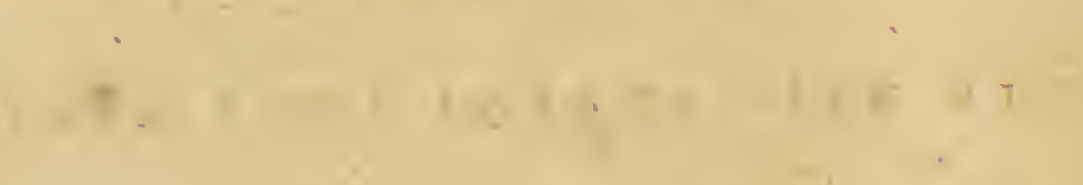

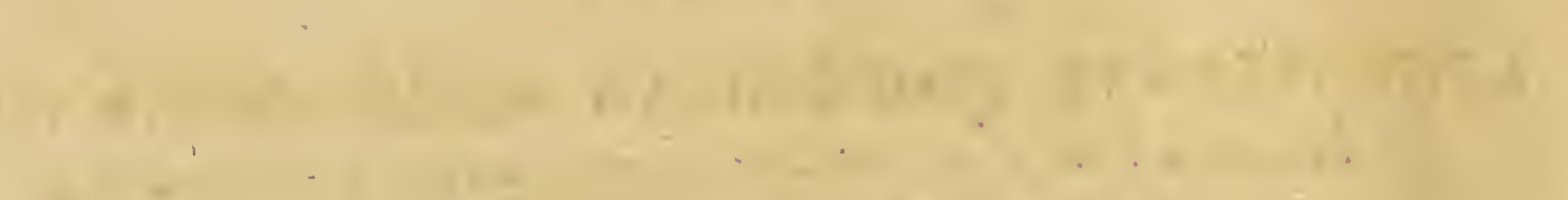

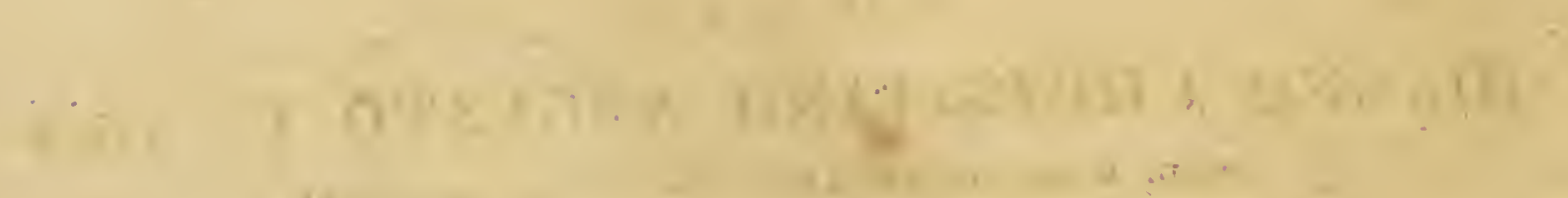

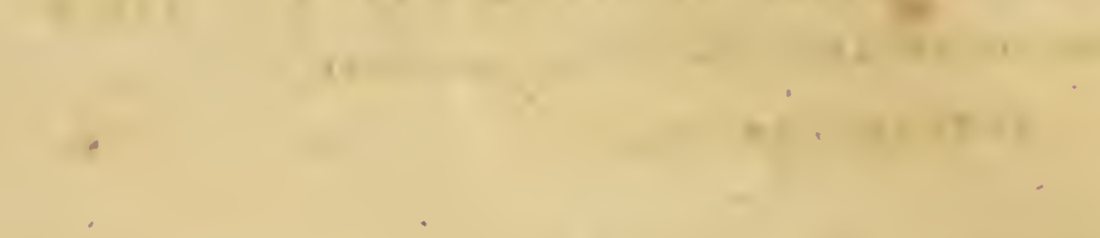

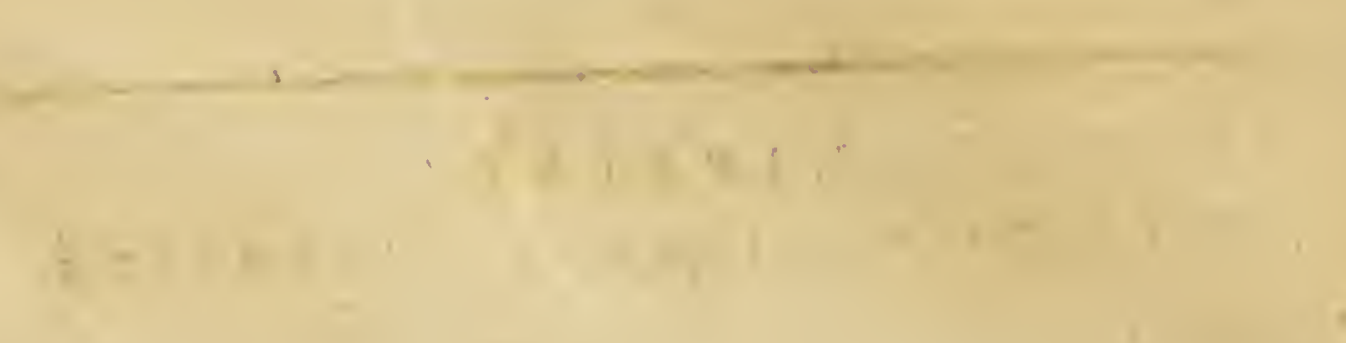




\title{
VIRO PRAENOBILISSIMO
}

\section{CHRISTIANO GODOFREDO SCHWAEGRICHEN}

\author{
II E R C A T.OPI C LA RISSI II
}

$\begin{array}{lllll}\mathbf{P} & \mathbf{A} & \mathrm{T} & \mathbf{R} & \mathbf{I}\end{array}$

SVMMO PIETATIS ET AMORIS CVITV

$$
\text { PROSEQVENDO }
$$

Hó LIBELLVM SACRVM ESSE VOLVIT

$A \quad \mathrm{~V} C$ C $\mathrm{T}$ O 


$$
29330
$$




\section{DILECTISSIME PATER!}

Quamvis Tibi, quem non' laudis et gratiarum actarum cupidum esse, muliis et variis modis compertus essem, mentis meae sensa, ex omni tempore, silentio obvelare magis, quam verbis palam facere coactus sim: hoc tamen loco mihi cavere non possum, ne Tibi, quis animus mihi sit litcris proponam. Scilicet, quum primum ad aliquid publice edendum accingar, nihil antiquius et sanctius mithi esse potest, quam ut eas studiorum primitias Tivivoveam, a quo, quaecunque in literis addiscendis hucusque profecerim, quaecunqne unquam profecturus sim, ea omnia me accepisse gratissimo animo referam. Nec quidquam. magis in votis est, quam ut Tibi, carissime pater, haec manca et exigua aliqua certe ratione probentur, guae etsi non satis digna bonis innu. 
meris, quae de Te in res meas largiter redundarint, esse confitear, tamén imperfectiora proponere malui, quam sensui diram moram diutius iniungere: Caeterum vitan et valetudinem. Tram, mihi exoptatissimam, summi numinis curae commendo, ardentissime precatus, velit. incolumen Te, et animi hiliuritate iusum, ad seram senectutem-conscrvare, ut multorum liberorum res prosperas a Te constitutas et fundatas esse, laete conspicias; idque laborum dulcissimum pramium non defore firmiter sperare audeo. Egुo vero ntt aniore paterno Tuo, et vitae morumque exemplo dignus evadam, hic mihi labor, hoc mith semper antiquissimum studium. extabit. 


\section{PROLEGOM ENA.}

Qumm multi sint, qui plantas quaerere, per partes examinare, describere, pro re satis quidem iucunda et facili, parum vero laudabili, vsuique communi vtili habere soleant: spes tamen nunquan decrit, fore, vt expositis et perspectis is, quae ad vitae communis promouendae vsum, ad bona et praestantia a nociuis et ingratis alimentis distinguenda, ad efficacia et salubria, $a b$ ineptis et periculurs vitae minitantibus medicamentis, seiungenda, botanica disciplina valeat: persuasio istis vel sponte oriatur, non spernendos esse, in rem herbariam locatos labores et industriam. Sed si multos opprobrio isto plantarum cognoscendarum studium, vtilitate oculos per se feriens, saepe grauare videmus, quid futurum insectorum curae et contemplationi diuinabinus, quae minutiarum studii, ludribiique speciem prae se ferre possit. Non igitur mirum, multis et variis modis saepe eos reprehendi, qui exiguorum et vilium istorum auimalium çuram gerant, locos natales 
investigent, corpora spectatui servent. Non tamen sempi. terna spretio eos vrget; nam stragibus horrendis per minutissima animalia casu exortis, silvarum vastis tractibus, quasi fulmine illato, torrefactis, arvarum laetis frugibus, ceu falcibus, abrasis, olerum splendidis frondibus ad virgultarum speciem exesis, exterriti omnes concursitant, dispersis cinerum nebulis, emissis venenoruny combustorum fumis, hostem ignotum evertere tentant, qui, ipsorum ignorantia fretus, dulci pace et quiete degit, genusque magis etiam propagat; usque dum per coeli asperitatem, aviua infestarum frequentiam, minutissimorumanimalculorum insidias ad interitum redigitur. Tum vero temporis luculentissime patet, non inutiliter otia trivisse, qui insectorum naturae cognoscendae studerent: et omnino confitendum est, ab hisce solis tuta et rei rationi convenientia remedia, dari, darique posse; quum. devastatorum istorum vilium tantum, tamque varium minetur agmen, cui fugando infinita remediorum varietate opus sit, si, finem assecuturos, nos gestare velimus. Nihilnminus non soli culpandi sunt, qui insectorum investigandorum studium spernere solent, sed illi quoque ipsi, qui entomologorum nomen laudemque sectantur. Eorum enim sunt, qui in eo acquiescant, insectorum aut corporeas varietates perspexisse, sut copiam pro systematum legibus in ordinem oomposuisse, 


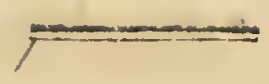

aut splendore et colore amoeno cubiculorum parietes exor nauisse; quae vero ad vitae victusque rationem pertineant, et quae luculente vitae communis vsui et ruralibus negotiis plurimum vtilitatis et cogitationi maxime notabilia, et iucundissima quaeque suppeditarent, ea omni modo negligere videantur. - Et rero si ad entomologorum scientiam nihil aliud poscendum est, quam insectorum aliquot imagines et staturam, quae rel adeo e sola pictura cognosci potest, imaginatione et memoria tenuisse, nomina didicisse: fatendum erit, aut istos non bene de disciplina sua mereri, aut hanc ipsum parum habere commodi et communis vtilitatis. Ne. que tamen ex altera parte reticere possumus, esse entomologiae studium, inprimis, si quis omnium classium habitum et indolem perscrutari tentauerit, et molestia laboreque plenum et ambitu infinitum; adeo vt vel botanicorum labores valde superet. Qnis enim est, qui non obstupescat fere, in Faunae Germanicae, quae dicuntur, initis, solorum insectorum coleopterorum duo fere millia specierum recenseri, eorumque numerum indies angeri; qnum tamen eorum agmen a papilionum copia forte superari, ab aliorum classium civibus fere aequari noverit? quis est, quin sordida et deserta loca, quibus insecta quaerere et obseruare, vel adeo domi suae nutrire oportet, intrare aut penes se pati recuset? quis 
est, quin coeli iniurias, aestatis ardentissimos calores, autumni supervenientes imbres, vernas inundationes periculosas, hiberna silvarum silentia tolerare, reformidet: accedente, otii cuius plurimum opus foret, penuria, praemiorum summa desperatione, valetudinis instante periculo. Quare vti ad eas, quas modo dixi, perferendas molestias, laboresque suscipiendos, peculiaris quaedam mentis alacritas et pertinacia, corporis eximia firmitas, necessario postulatur, quae neque intenso literarum studio, neque vitae sedentariae veluti carcere hominibus comparatur: ita literarum expertes, in insectis colligendis, educandis, dignoscendis, saepe felicissime versari obseruatur; nec raro dolendum est, eosdem ignorantia sua impediri, quominus ad altiora sese elevent, et earum rerum memorabilium, quas fieri observarint, causas inuestigent, comparatione plurium et inter se, et cum iis, quae aliis animalibus eueniant, et quae iis propria corporese facultatis sint, ad generalia de physiologia et philosophia componenda procedant; tum verecundia deterreri, vt ea quae ex ipsius naturae contemplatione didicerint, publice enarent; a viris doctis vero omni modo vili haberi, non melius etoceri, vbi errarint, neque satis de scientia sua examinari et interrogari; rnde sacpe conqueri multus audimus, quod, qui corpora viuentia perscrutentur, non scribant, qui scuibant, non perscrutati sint. Ea- 
que ipsa inter-causas est, propter quas, etiam in Germania nostra, quae alias terras entomologorum frequentia omnino superare videtur, saepius insectorum formas describi legamus, quam eorundem habitum et indolem enarrari. Qua in re etsi insectorum species, antequam rite a similium turba accurate distinctae et extricatae sint, tute et subtiliter obseruari non posse, eosque, qui earum diuersitatem exponant, de studio suo bene mereri, nullus nego: tamen etiam in is speciebus, quae satis notae sunt, vitae goneris et metamorphosium rationem saepe nimis negligi, repreliendere audeo; quod quidem negligentiae documentum, etsi in systemate conscripto, spatii exiguitati largimur: in enumerando tamen insectorum, cuiam regioni indigenarum, copia studiosilis vitari, et loci opportunitas suppeditat, et vero etiam rei ratio exigit; id enim nobis postulare possumus, vt, qui animalcula quaedam se collegisse profteatur, eorundem locum natalem, habitum, consuetudinem, simul obseruata, proponat. Et hoc ipsum est, quod vtilem et cogitationi dignam et acceptam, istarum minutiarum perscrutarionem reddere valeat. Sic enim augentur physiologiae vninersae capita; illustrantur, quie similia aliorun animalium ipsiusque hominis naturae insecta habeant, quae dissimilia; indagatur via, qua devastationes immensae procuzentur, qua ad nostra commoda operari, inscia insecta docean- 
tur, qua ad morbonm impetus infringendos, inter medicamina efficacia recipi queant. Iliud autem, commodis plenum, optinie naturalis historiae amantes tum efficient, quum ab exterorum insectorum, magnitudine et venustate praepollentium, collectione se abstinuerint, inque terme patriae incolas oculorum obtutum converterint: quid enim iurat, multorum insectorum longam seriem mente, nominumque longas lineas memoria percepisse, si, quae nobiscum habitant, dumnaque inferunt, neque cognoscere, neque prudentia vincere didicerimus. Rectius itaque civibus operam nayabinus, si agri nostri incolas. recensemus: eorum vero describendorum non aptior facile locus invenitur, quam fannae entomologicae titulus; nam in systematicis libris non sufficiens permittitur, spatium. Ibi vero constituendum erit primum, quae insectae adsint, deinde, quem victum sibi quaerant, postea, quod vitae genus gerant, tum, quae noxia vel quae commoda hominibus comparent, tandem, si hoc addere lubet, num vires morbis medentes habeant, et qua ratione applicari possint. Quod victus rationem cuique facile botanicae disciplinae necessitas et arctus nexus elucet, nec potest entomologus dici, qui non certam, etsi quovis modo acquisitam, plantarum multarum notitiam calleat; nec vero etiam botanicus, inprimis in loco de plantarum morbis, bene proficiet, nisi in. 
sectorum certe aliquam scientiam acquisiverit. Quare commodissimum omnino visum est, utramque disciplinam ita iun. gere, vt plantarum et denique insectorum turbas enumeremas, praemisso de soli, regionisque reliquo habitu, sermone; nam et plantas sponte crescentes et animalia is victitantia, soli, illa producentis, haec recondentis, mira quaedam conuenientia intercedit. Scilicet infitias nemo ibit, insectorum specierum varietatem pendere ex arhorum plantarumque natura, quarum quaelibet sua pericularia nutrire solet: sed hoc etiam expertum satis est, quamque terrae mixtionem suas peculiares habere plantas. Ita necessario sequitur, in regionibus calcareis alias, in argillosis alias, in arenosis alias plantas progerminare, alia insecta degere. Sed ne de plantis quidem omnibus satis exploratum habemus, cui potissimum humo quaelibet innasci soleat, quamque in eo, aut alio, minus sibi conveniente, =formam assumat, etsi ea ad physiologiam et pathologiam plantarum, et ad geopraphiam botanicam, et ad oeconomicas medicasque rationes plurimum valere appareat. Neque tainen ea res, si modo attente et studiose rerum naturalium curam -agimus, inventu difficilis est. Quum enim per regiones variae indolis itinera fecimus; ex omnium, quouis loco inuentarum, plantarum, habitusque differentiarum perspectarum, complexu, fingitur nobis, fere nescientibus, quaedam quasi soli cuinsque 
uniuersa notio; qua in plantis mire adiuuamur ita, vt, solo fugitiuo agri alicuius inspectu, possimus quasi praedicare, quaenam ibi vegetabilia, et quanam conditione incenturi simus. Neque caret hoc vtilitate communi; si enim probe tenueris, quem habitum in solo proposito planta quaedam, verbi causa fruges, nactura sit, aut quae plantae spontaneae fruges obtecturae; vel arbores exhausturae, àut particulas nutrititias crepturae sint; tum et humi varie instituta laedificatione, et tempore, quo inimicae accrescere solent, cognito, eas interimere, aut alio modo tollere, felici successu conaberis. Iam etsi, quam plurimae plantae, certe Germanicae, glebarum mixtionem malint, quam in quoque solo speciem induant, notim sit, supersunt tamen multae, de quibus ista nos adhuc lateant. Sed quod insecta attinet, multa nobis ignorantur; nam haec vel illa in arena fodere, alia in solo pingui enasci, scimus: sed num pro diuersa plantarum, fruticum, arborumque eiusdem speciei natira, diuersae species insectorum adsint, vel an eadem species ratione ciborum, per soli mixtionem in plantis aliter comparatorum, variare soleat, id plerumque ignoratur. Cuius exemplum forte est Luicanus Ceruus, in quercu maioi, in salice multo minor; est hic igitur varietas ratione plantae: alia specierum pro climatis solique, ratione et, inde dependente, plantarum structura non desunt, 
si, in montosis, et australioribus species insectorum natas, nostratibus tiusdem nominis maiores et splendidiores evadere docemur. Quod praeterea capita historiae naturalis, de quibus loquimur, attinet, superest, ut fateamur, latere: num species quaedam insectorum pro diversa arborum solique, eas ferentis, habitu, diversum vitae genus agant, quaenam in quadam agri conformatione maxime noxia, et quae minus sint, in quo regionum genere virium medicinalium plus in se ani. malcula recondant. Ea vero pleraque ita exquiri, literisque mandari posse reor, ut, quicunque Faunam Floramve scribat, praemittat quaedam de terrae suae habitu et natura, deinde cuique speciei animalium et plantarum notulam adiungat quae eius locum natalem designet, et victus rationem indicet, et num frequens ista àut parca adsit, moneat, Quod si propositum viris, scientiae naturalis peritioribus, non displicuerit, equidem eiusmodi topographian studiose elaborare suspicerem; iam vero, exempli causa, topographiae Lipsiensis primas lineas ita describam, ut praemissis de universa soli nostri conditione quibusdam, descriptisque locis Florae et Faunae memo. rabilibus, plantisque aliquibus, quae loco cuique maxime peculiares, et, ad eius indolem idea concipiendam et tradendam, aptissimae videantur, nominatis: ad specialiora transeam. Ad integram regionis topographiam naturalem pertinent loco. 
rum omnium descriptio, lapidum, plantarum, animalium omnium definitio; coeli, ventorum, climatis, fatorum narratio; et omnium, de vegetatione, victu, et oeconomia animalium et plantarum notandorum, epitome; qualem integram quum hic exhibere nec velim, nec possim; quaedam sola eius capita digito monstrabo; et quum, virorum clarissimorum, Boehmeri, Schreberi, Hedwigii, et Baumgartenii opera, de florae nostrae civibus sufficienter expositum sit, eorum tantum aliquos nominari satis esto: animalium maiorum et vermium historia, quim aut plenius exposita, aut summis difficultatibus implicita sit, plane praetermittetur: insectorum vero catalogi initia afferentur. Sed quum arbitrer, instituti mei rationem, iis, quae mox expositurus sim, satis apparere, et quum loci opportunitas, longae nominum seriei proponendae parum favere videatur; iam catalogum istum in aliud tempus servare invitus cogor. Caeterum lectorem de meliori monitum velim, esse hoc meum conamen, nil quam periculum, quod num abiiciendum; an amplius resumendum, tempore maturatumi, industria expolitum repetere ausim, peritiorum iudicio dependebit. 


\section{LOCORVIM DESCRIPTIO.}

Agri nostri habitus talis est, quem, primo intuitu, plantis multis et rarioribus, insectisque generandis non favere perhibueris.' Est enim ei magna planities superficiei, silvarum exigua varietas; desunt montes herbidi, silvae vastae, saxa frequentia, rupesque solidae, nisi paullo longius excurrere velimus. Arvorum cultura diligentissima, si, lactissimis frugibus pomorumque splendore, ambulantium oculos delectare satagit: insectorum simul amanti, plantarumque spontanearum cupido spem infringit, labores illudit. Qua de re videmus quotannis hoc vel illo loco plantulam dilectam diro aratro eversam; insectum, sede et domicilio deperdito, oculorum conspectum et observantiam fugisse. Sed guis rusticanis hortulanisque hoc in opprobrium vertet, quum terrae natura ipsos magno. pere ad labores invitet? Est enim solum maxima e parte fertile laudisque plenum; constat nempe terra luto, humoque pingui commixta, hic illic, inprimis in elatioribus campis; arena- distenta. Lapides substrati; in aliquibus locis, vt prope pagos $Z$ schocher dictos, nudi prodeuntes, argillosi generis sunt et argillae vulgaris induratae Werneri nomine veneunt; 
paullo remotius versus orientem saxum porphyrium, versus meridiem sàxa arenaria et calcareus aequabilis, qui versus occidentalem plagam continuatur, et in nonnullis locis gypsa. ceam naturam, verbi causa prope Dürreberg, pago ob salinas electorales perquan celebro, induit, inneniuntur. Quae alia lapidum genera occurrunt, granites, silices pyromachus, petrefactis saepe distinctus, comeus, Feldspathum, Quarzum, et schistosus is, qui lapis Lydius vocatur, aliaque; ea non terrae huic innata; sed per aquarum cursum invecta esse videntur, trituque diuturno angulis orbata. Neque abundat regio lapidum, in arvis et pratis proiectorum, profusione, quare difficiliora carabi staphilinique et consimilia genera investigatu, sub humo latent. Saxorum vero muscis tectorum ct umbrosorum, coleoptratis ditissimarum latebrarum, inopia est; nisi quod prope Grimmam aliquid rupium, nec tamen entomologiae satis aptum, conspiciatur. Adsunt vero prata humida, spongiosa, turfosa, maxime iuxta Partham fuvium, ibi muscorum, aliarumque plantarum paludosarum abundantia vestigia pristinae, quae nostrae regioni fuit, indolis satis ostendunt: paludes olim totam plagam septentrionalem occuparrunt, ut ii ipsi loci, qui a cerdonibus habitantur nomenque gerunt, olim stagnis repleta fuerint. Ibi sunt insectorum arjuaticorm agmina. Versus occidentem videmus pratá sil- 
vasque undarum impetui obnoxiä, per veris magnam partem fossas et depressiora loca, aqua stagnante plena, foenisecii spem saepius fallere; entomologo laetam dyticorum messem comparare.

Vegetabilium autem ea natura est. Prope urbem, qui loci humido, lutoso, depressoque solo sunt, laete provenire gaudemus quercus, carpinos, acer campastre, tilias: betulas, fagosque et pinus frustra quaesierimus. Quamobrem nemora haec umbrosa, humida, muscorum pulvinaria, levocoii, alliorum, primularum, graminum plurima ferre obseruatur; suntque iisdem propria veronica montana, quae alias vix, nisi locis valde montosis, occurrere solet, bromus giganteus, euphorbia dulcis et palustris, phascum patens, Leskia attenuata, fissidens bryoides copiosissimus, hypnum alopecurum, nec non fungorum inprimis sphaeriarun permulta; pratis vero contentis veronica longifolia, thalictrum flavum et similia. Hac situs ratione exposita, nemini non incidit, adesse nobis insectorúm, paludibus farentium, copiam, tipularum, culicum, ephemerarum, phryganearum, phalaenarum et inprimis noctuarum, quarum erucae plantis supra nominatis vescantur; verbi causa: noctuae Fimbriae, Ianthinae, Pronubae, Orbonae, pulmonaris, $L$ albi, pallentis, aiiorum. Coleopterorum vero inopiam conquerimur; desunt carabi maiores, si granulatum 
Linn. excipias, et multa alia, çuorum nomina recenseri longum foret. Et alia quoque causa est, quae harum silvarum divitias imminuat; ea, quod lingna et trunci putridi rarius inveniantur, et quod aqua, Auviis egressa, multa nobis auferat. Huius quidem indolis omnia ista nemora invenimus, quae Plissam et Elystrum, fuvios, comitantur. Alia vero omnino res est, ubi ab eorum fluviorum tardo et obscuro cursu utrinque ascenderimus ad loca altiora; tum enim mox invenimus solum siccius, pyromachorum frequentiam, nemora arborum varie. tate, plantarum novitate, insectorum agminibus praepollentia; adsunt betulae, pinus, rarius tamen figi et abietes. Habemus ericam, airam flexuosam, spartium, genistas, pteridem, polypodia maiora, trichostomum pallidum, lichenes crustaccos, terrae instratos, et, inter insecta cerambyces, buprestides, cicindelas, bombyces versicoloram, V nigrum, bicoloram, fagi, Milhauseri, noctuas matronulam, fraxini, augurem, gryllum coeruleum, hemerobium phalaenoidem et similia. Quare non peccare mihi videor, si omnes regionis nostrae partes ita in duas classes dispescam, ut alteras pingues, alteras macras nuncupem; quam utramque classem rursus dividerem in umbrosas et apertas. Vmbrosi sunt loci silvatici aut arboribus tecti, aperta vero prata et arva; nam etsi paucae tantum plantae et insecta mere arvicolae et praticolae sint, videnrus tamen et haec 
quendam delectum facere. Scilicet arva aperta pinguia largiuntur phascos subulatum, crispum, dicranum Schreberi, carabum ustulatum, elaphros aquaticum, riparium, Alavipedem, aliosque: arva vero macra phascos ostendunt copiose piliferum, muticum, dicranum purpureum, hypnum albicans, iisque supersilientem panorpam hiem?lem, scaritem arenarium, cicindelam Germanicam, carabum auratum frequentissimnm et alia. Quodsi vero etiam in macris, siccioribus, elatioribus locos offenderimus humidos, eorum tamen indolem a depressorum habitu ita differre intelligimus, ut plantarum inveniamus diversas species, carices hirtam, aliasque huic solo proprias, potentillam supinam, spergulam nodosam, anthocerotes, blasian, Ricciam glaucam, minimam, agaricos, et insectorum elaphros riparium, paludosum; elophoros minores; in umbrosis autem; caricem tomentosam, brizoidem, iungermanniam bicuspidatam, et aliam, ei similem, minorem ignotam, multifidam, lichenem horizontalam, caninum, agaricos plures, carabum hortensem, Inquisitorem, parumpunctatum, holosericeum.

Sed ut agri nostri habitus plene et perfecte describatur, necesse est, singula, quoque loca depingi; quem, si quis, recte teneat, naturae curiosus, aliam regionem ingressus, statim intelliget et comparatione colliget, quibus modis haec 
terra a patria sua differat, et quibus eiusdem similis sit; qua ibi inveniri possint, quomodo inquiri debeant; oeconomus: quibus inimicis dimicandum, quomodo dimicandum sit. Quare nunc singula enarrabo initium a propioribus facturus, et breviter quasdam plantas et insecta, iis peculiaria eorumque naturam indigitantia enumerabo, caeteris, quae simul communia aliis quoque locis adsunt, quippe in catalogo meo nominandis, neglectis.

Est pratum in plaga occidentali, die Vogeheiese dictum, solo duro, limoso, lapidibus carente, gramine dense irretito tectum; carduis lanceolato, polyacantho, crișpo, serratulis arvensi et tinctoria, geranio pratensi, salicetis, plintisque ruderata amantibus, fertile; depressum, inundationibus obnoxium, stagnulis interstinctum, vere plenis, aestate ex parte exsiccandis; sed plantis palustribus, (excepta arundine, typhis, alismate, caricibus, laete vigentibus) muscisque, praeter fissidentem bryoidem, collemata aliquot, etiam lichenibus carens. Ibi mirae dyticorum et hydrophilorum, praesertim minorum, heteroceri, donaciarum, hemipterorum aquatilium turbae; papilionum maiorum et minorum caritas, nevropterorum et dipterorum frequentia. Similis naturae sunt prata maiora silvarum, ripas Plissae et Elystri Auviorum comitantium, qua- 
rum tamen prata minora et tramites muscos et plantas, umbrosa amantes, alunt.

Diversa ab his maxime sunt, prata, Partham rivum am. bientia, quippe muscis palustribus, hypno nitente, cuspidato, scellato, Lcskea dendroide, mnio cuspidato et similibuś, caricibus plurimis, chara vulgari, acoro Calamo, salice capraea aliisque, carduo palustri, conico oleraceo, comaro palustri, iunco filiformi agrostide Calamagrostide, aira coerulea, caespitosa ditiora, humo pingui, nigra farcta; per totam aestatem subhumida, vere spongiosa, dyticis non adeo abundantia, sed zygaenis minoribus, papilione-Hylle, Typhone aliisque plebeiis ruralibns, bombyce falcula, saperda cardui, noctua $L$ albo, furcifera, aliisque similis indolis, ditissima.

Vicinus locus est iuxta rivulum dis Riizschke, qui poetarum ambulacri nomine venit. Ibi populi nigrae, salicum copia, olerumque laetissimă arva, humo pingui nigra, adest ribesii nigri aliorumque virgultorum quantitas, multae alticae, aliaque agricolis infesta animalcula, geometrae, noctuae, Sesia tipuliformis occurrunt.

Silva urbi proxima non fusius describenda est, quum eius habitus ratio supra data sit; loco de silvarum urbi proximarưm indole. 
Sed alius nos tenebit locus, Faunae et Florae gravissimus; collis nempe nemore tectus, qui Bienis vocatur. Hic locus, etsi collis nomen vix mereatur, quum parum elevatus sit, omnia habet, quae in montosis occurrunt. Situs est versus occidentem iuxta viam publican Martisburgeasem; e campis siccis arenosis, septentrionem versus, leniter ascendit altius, paullo magis praeceps decessurus; occidente prato per fonticulum irrigato, septentrione prato, alneto, campis humidis cingitur. Multas arbores in vertice alit, quercus, carpinos, populum tremulam, betulas albas, prunum spinosam, oxyacantham, tilias cordatas, pancasque pinus silvestres satas alit, et vero plantas, quae in aliis agri nostri regionibus non reperiuntur et proprie monticolae sunt.' In colle, qui siccus et ex parte umbrosus est, inveniuntur hypericum montanum, vincetoxicum, antherica liliago et ramosum, phytema spicsta et hemisphaerica, silene nutans, Jychnis viscosa, galium silvestre, silvaticum, rotundifolium interdun, coronilla varia, cistus helianthemum, pulmonaria angustifolia, primula offciralis, Ilium Martagon, Scorzonera himilis: in colliculo et agris orientalibus, anemone Pulsatill, festuca myurus, aira canescens, festuca ovina, polycnemum arvense, carex humilis, montana, praecox Schreberi, scabiosa ochroleuca, ornithopus perpusillus, ;orchis Morio, sambucina, cladonia rangiferina, 
papillaria et similes plantre. l'aucom passum intervallo, occidentem versus, solum in pratuin deprimitur, fonticulo sulcatum, ad cuius originem plantae paludosae, carices per-. multae, festuca arundinacea, spiraea filipendula, hypnum scorpioides et filicinum laeta exstant. Pratum, aquulae istius propagine cinctum, versus septentrionem abit, exhibet Cirsium Halleri, sive varietatem cardui acaulis, tussilagines hybridam ct Petasiten, lotum siliquosum, gentianam Pnermonanthen, laserpitium Prutenicum, Peucedanum officinale, Iridem Sibiricam, ophioglossum vulgatum, in agris, prati ditioni subductis, vicinis, antirhinum Elatine, spurium, minus et guae sunt reliqua. Hic igitur, loco, qui uno horae quadrante, concitato passu, permeayi posset, plantarum summa varietas est: non mirum igitur insecta plurima ibi oculo offerri, inter quae, catalogo taediose, longo suppresso, notare lubet, chrysomelas, cistelas, melolonthas et alia, de quibus in catalogo insectorum Lipsiensium dicendum, gryllos, cimices, cicadas inprimis auten tinearum et pyralidum Limaci miram copian et diversitntem dari. Proprius vero huic loco papilio plebeins ruralis quidam, et papilio silvanus, carabus haemorrhoidalis, cassida nobilis ct margaritacea, noctuae augur, bombyx $V$ nigrum et alia.

Laudania vero pyeprimis silva, quac Acatemica dicitur, est; in qua, etsi plantarum non tanta sit varietas, nonnullae $\mathrm{C} 2$ 
tamen rariores occurrunt: trientalis Europaea, stellaria uliginosa, bromus asper, elymi, sanicula Europaea, pyrolae, vaccinium Vitis idaea, serapiades longifolia, latifolia; polypodia Filix mas et femina, lycopodium clavatum, Buxbaumia foliosa, trichostomum pallidum et alia. Arborum vero haec ratio est, ut, quae silvae pars urbi propior sit, quercubus, populis tremulis, betula alba, tiliis, carpinis, corylis, paucioribusque fagis componatur, sitque silva in marginalibus sicca et arenosolutosa, media vero lutosa et umbrosa; contra versus meridiem: populus tremula et betula alba, inmistis paucis pinubus silvestribus, et abietibus, praedominentur, solumque siccius et erica ubiqne tectum. Ea soli, situs, arborumque varietate, totiusque trăctus vastitudine tanta insectorum copia fovetur, quantam exspectari vix liceat; omnia enim, quae in silvis Lipsiae propioribus vitam degunt, simul cum aliis plurimis in hoc unum quasi receptaculum congeruntur. Sunt hic coleoptera plurima, de quibus alio loco agemus, lepidoptera fere plura; propria vero sunt noctua Matronula, bomby: bicolora, rosea versicolora, cossus 'Terebra et permulta alia.

Neque plane praeterire possum pinetum, quod urbi proximum est, et ad pagum, nomine Lindenthal, iungitur, quippe guia plura sibi peculiaria recondat. Est nemus mediae magnitudinis, campis siccioribus circumdatum, solo âsperiore, du- 
riore, lutoso; pinus silvestres continet immistasque quercus, populos, betulas, salices. Plantas paucas fert, silenem nutantem, phascum nitidum, hypnum Cristam castrensem, lichenem physodem fructificantem, et airam flexuosam, spartium aliibi nobis rarum; continet insecta pinicolas, sphingem pinastri, bombycem pini, noctuam piniperdam, phalaenam piniariam' tenthredinem pini et alia et praeterea multa, quae in silva Academica occurrunt, etsi minori copia.

Praeterea entomologiae aptae sunt viae publice, populis sacilibusque refertae, vbi callidium salicis, cossus ligniperda, sesia apiformis et asiliformis, tipula pectinicornis et alia nascuntur: neque minus latudandi horti suburbani; inter quos inprimis Bosinus, ab arte neglectus, multas plantas spontaneas, verbi causa fumariam cavam, capnoidem, antirrhinum Cymbalariam, helleborum hienalem, digitalem ferrugineam, Scillam amoenım, ornithogalum nutans et alia profert, et coleoptera plura, noctuas Finbriam, Pronubam, Ianthinan, bombyces ponaria habitantes, papilionem Prorsam et Levanam, vesparum, crabonum, apum, philanthorum, muscarum et syrphorum multas species alit.

Sed ne remotiora plane neglexisse videar, dicendum erit de oppidorum vicinorum agro; in eoque me non errare arbitror, dum ad Faunae nostrae ambitum eos locos transferam, 
quum in Floris Lipsiensibus eorum saepe mentio fiat, sintque ipsi satis vicini, ut ad eos iter reditumque uno die emetiri queamus. Sed de his, praesertirn entomologica ratione, paucis agendum erit, quum et libeili huius conditio brevitatem exposcat, et me istos agros propter distantian ab urbe, laboribus academicis impeditum, non ita studiose et frequenter percurrere, ac opus fuerit, potuisse doleam.

Leucopetram quae via tendit, in ea mutatio agri plani et depressi in collinum conspicua est. Solum sensim eam pinguem et tenacem indolem, coloremque nigrum induit, quibus vicinae Thuringiae laetissima arva superbiunt, estque in siccioribus durissimum in depressis spongiosum, et si Numburgum iter continuatur, calcareos lapides argillosos excipere observantur. Silvae e quercetorum umbris in mixta pinuum, fagorum, quercuum, abietum, plantaria immutantur é insecta et plantae monticolae apparent, ut andropogon Ischacmum, reseda lutea et luteola, psora lentigera, chrysomela cerealis. Lamia fuliginator et alia.

Leucopetram et Martisburgum inter locus botanophilis gravissimus pagus Koctschau est, vbi solo, per salinarum culturam frequenti sale impraegnato, plantae multae maritimae proveniunt ut, aster Tripolium, glaux maritima, plantago-maritima et subulata, Scirpus maritimus, chenopodium maritimum, Samolus Valerandi, et alia plura; insectorum rero non tanta fertilitas est. 
Martisburgi ager planitiei Lipsiensis similis pauca sibi propria habet, nisi hippurim vulgarem; solum pingue silvaeque umbrosae quercubus, alnis, et similis naturae arboribus refertae. Sed versus occidentem et septentrionem incipiunt colles in montes hercynicos abituri, qui plantarum rariorum fertilissimi sunt; et in Flora Halensi a Leysero conscripta, Mansfeldiae nomine saepe alleguntur; astragali exscapus, arenarius, Danicus, pilosus, cynosurus duris, euphorbia Gerardiana, marrubium supinum, Creticum, aliaque; insecta vero plurima, quae Halensia dicuntur, sedes ibi et domicilia habent.

Hala Saxonum ad Florae nostrae campum non duci solet, etsi non adeo remota, quoniam alienae ditionis est: sed quum in Flora Halensi a Leysero conscripta, planta quaedam, comarum palustre, recensita sit, cuius non alius locus, quam paludes prope Schönfeld, pagum, urbi nostrae valde vicinum, indicetur, possemus nos quasi res repetere et plantas Halenses nostris adnumerare.

Ad Halam usque continuantur silvae, ad Plissae Elystrique ripas eodem habitu, quo Lipsiae sunt eademque continent. Plaga reliqua nostra septentrionalis, quippe frugibus lactissimis obtecta, nemóribusque orbata plantarum nihil memorandi profert; sed prope Bitterfeld silvae et betuleta vástiora incipiunt; quae ad silvas Anhaltinas se adiungunt, plantisque 
et magis etian insectis certo ditissima, etsi nondum satis perlustrata. Delitzsch oppidum est agro nobis parum apto; Me. morabilius vero Düben, silvis vastioribus soloque arenoso cingitur; satis liquet, regiones sabulosas, si modo silvis abundent, multa alibi rariora gignere. Agri huius divitias, quum anno praeterito, nou tardo satis passu, permeabam et quoad insecta, et quoad plantas, de quibus alio loco dicetur, summas esse, ita perspexi, ut, non esse mihi eum lustrandi otium, valde dolerem

Versus orientem pauca propiora notabilia habemus, excepto colle templi Sanctae Theclae, qui in Schreberi spicilegio saepe allegatur sub agrostidis vinealis, osmundae lunariae, alyssi incani, seseli saxifragi, caricis montanae, saginae erectae mentione et silva der Wilwisch dicta, quae arborum copia mixtarum entomologo aliqua, botanico vix aliquid offert; sed prope ea, in agro Baunsdorfiano parvum pinetum est, bombyce pini et noctua piniperda saepe male abundans. Si rero in ea plaga continuatum est, pervenitur ad silvas betula alba et pino silvestri, sed aliis quoque arboribus refertas prope pagum Machern, quae insectis ditissimae sunt. Extenduntur versus meridiem ad Brandis et Polenz ita, vt versus Muldam Aluvium vastiores sint, solumque colliculosum obtegant. Ei regio plantis et insectis generandis omnium nostrarum aptis- 
sima propter longiorem distantiam iusto tempore nondum satis percursa est; sed in Oehlmanni; noto papilionum et peritissimo inquisitore, cistulis, qui quotannis expeditionem quasi, in illam regionem molitur, praestantissima quaeque inventa esse miratus sum. Habet eadem regio hoc peculiare, quod porphyria strata recondat; versus Grimniam vero Muldam ripibus, argilla vulgari indurata constantibus, cingat, quarum cacumina nemoribus betularum, quercuumque apte nobis teguntur. Tractus isti silvosi procedunt ad meridionalen plagan usque ita, ut prope Nounhof depiessiores et humidiores sint, pinubusque magis abundent, elatioră vero postea petant et versus Coldiz solum colliculosum investiant ramosque usque ad silvam nostram Academicam porrigant, cuius incolas nobis dilectos, haud dubie illis debemus. Adnumerari iisdem silvaticis potest nemus pagum Steinbach circumiens, quod valde memorabile et locus nostrorum proximus cst, qui phalaenam Tau alere soleat.

Etiam Zizensis ager non debet negligi, quippe nemoribus refertus, soloque pingui maxime fertili ornatus; collibus interstinctus, quorum interiora mox calcaream naturam induunt, quae, versus Geram, exteriori montium figura et coma manifeste oculos ferit. Continuantur ad Zizam vsque silvae Plissae et Elystri comites, codem, quo Lipsiae sunt, habitu; Zizen- 
siumque agri indolem magis variam reddunt, cuius et ipsius divitia Lipsiae nimis ignorantur. Ziza si ad Lipsiam tendimus, propè Zwenkau nemus iucundum, der Horst offendi. mus, silvae Academicae simile, etsi ambitu minus: si vero ad occidentem converterimus, per arva fertilia, studiosissime culta, itinerati, ad Leucopetram redimus, unde supri digressi sumus.

De cocli et tempestatis varietatibus, aliisque, quue in vitam et propagationem pilantarum et insectorum efficacia sunt.

Quod ad coeli temperiem et caloris, frigorisque vicissitudines attinet, notandum illud videtur, constantiam valde desiderari. Mox enim nebularum caligine vernalium et autumnalium, mox ventorum impetu, mox solis aestivi radis perturbamur, its tanen, ut aestus modicos, nebulas vero, pluviososque et ventosos dies frequentiores et molestiores habeamus, etsi imbribus, grandine tt turbinibus, quae in confiri Thuringia saepe furiant, plerumque nobis parceatur. Quae quidem res florae nostrae haud exiguo fructu est. Si enim siccitate solum maximam partem lutosum exuritur, pereune 
plantarum radiculae, glebarum duritie suffocatae; uruntur muscorum sedes, duro cibo insecta debilitantur; si vero imbres et pluviae effusae ceciderint, solum ita contunditur, ut, areae instar induratum, fruges depauperatas ferat. Sed rarius aestum, quam coelum turbidum et imbres conquerimur, eaque causa est, quae minimarum plantarum fructum et propagationem tweatur, quae silvarum raritate et tenuitate, umbrarumque deficientia mox periturae essent. Itague muscos florere nobis gaudemus, qui in aliis regionibus, silvarum vastitudine nostras haud praepollentibus; nunquam fructus ferre accusentur no. bisque gramina et aline similis naturae plantae serventur, agricularum nunquam remittente labore interiturae; cuius testimonio afferam Leskiam dendroidem, attenuatum, hypnum squarrosum, stellatum, filicinum; quorum posteriora florerè, etsi non semper fructus maturare videmus.

Sed observanti tamen firmiter persuadetur, et hac in causa, floram iacturas perpessam esse. Nam, exempli gratia, prata Schönfeldica iam non largiuntur splachnum ampullaceum, alneta eiusdem loci carent lichene pulmonario, iungermanniis et aliis aliquot; cuius causam pratorum, diligenter per-fossam exsiccatorum et terrae per ventos ex agris elatioribus vicinis advectae copiam subesse, Hedwigius, praeceptor summo sem- 


\section{$\square$}

per desiderio revocandus, docuit, atçue similia, si dicere fas est, infortunia, florae in posterum minantur.

Alia res, qua' Florae cives mutentur, exstirpentur, augeanturve, est crebra cxundatio aquàrum; quotannis vere silvae et prata fluviis adiacentia libidinoso aquac torrente teguntur, nonnunquam altiore ct pagis villisque pericula, minante. Quae, undarum vis ut multa nobis semina attulit, quaruin soboles, "monticolas, in plano nostro agro provenire miramur, ita alias interdum nobis abripit; aut eorum locos ratales ita mutat, ut vix amplius victum istae inveniaist. Et magis etiam et luculentius aquae efficacia in insectorum historia patet. Insecta enim, corio duriore tecta, afferuntur, vitáinque natatu mire servatam, hac in terra, posita prole, condunt; cuius quidem rei exemplum maxime in dyticorum genere est. Caeterum mirandum est, insecta perfecta coleopterorum, aut pupas lepidopterorum, etsi íqua diu circumlatas, vitam tamen, servare; nam pupae, quae glacie adeo inclusie inventae erant, felici fato in lepidoptera completa accreverunt. Et vero etiam erucas, aqua ablatas, migrare, hoc mihi exemplun fuit, quod aliquando in silva dem Rosenthale larva bombycis $\mathrm{V}$, nigri inventa sit, quae ibi nunquam occurrit, et ex aliis silvis advecta esse debet. Non omnis igitur oeconomo in aquae, litora transgressae adminiculo ponatur spes, nam insecta in- 
imica, forte per septimanam integram aquae immersa, tamen ex parte evadunt, idque etiam in aliis animalium ordinibus evenire, nuper edoctus sum, quum, post copiosissimam exundationem, in prato, per sex dies undis tecto, vix regressa aquae vi, talpas copiose fodere conspicerem.

Alia ratione Flora et Fauna per ventos locupletatur, ut erigeron Canadense, plantam, in America natalem, iam ubique ngri Lipsiensis crescere, nullám causam nisi ventorum impetum accusamus, qui, semel illata semina, ubique sensim sensimque circumegere. Idem quoque insectis, etsi rarius contingere notum est. Nec vero per ventos plantarum aut insectorum species plane perdidimus; insecta, leviora inprimis saepe ventis ab uno loco in alterum transferri, notum est, exstirpari vero non facile possunt, quum multa semper tardiora loco natali remaneant.

Alia Florae mutatio existit per coeli temperiem; namque aliis annis laete omnes plantas crescere videmus, aliis plurimas mancas et enarcidas; nonnullas adeo desideramus. Ita elatinen Alsinastrum, quae ante multos annos a beato Hedwigio in stagno prope pagum Lindenthal detecta, e decem annis nunquam iterum visa est, anno millesimo septingentesimo nonagesimo quinto eandem ipse copiosam ibidem inveni; sequentibss vero annis ne ramulus quidem apparuit. Ea vero in re 
inprimis nivis aut frequentiam aut incpiam plurimum valere, maxime plantae minores, cryptogamicae, docent: similia insectis quoque contingere elucet, quorum vero causae onnino incertius designantur. Nam si qnis per humorem, per annum aliquem saepissime cadentem, insectorum catervas dispersas esse diceret, obstat aliorum annorum historia, quibus, post crebras pluvias insectorum cohortes frequentissimae prodierunt.

Sed plerumque insectorum turbae minuuntur causis plane ignotis, quae tamen a tempestate pendere debent, licet plerumque non conspicuae. Sed obstat experientiae firmandae validissime nobis, specierum insectorum copia victusque varietas, qua fit, ut quae aliis animalculis noceant, eadem aliis prosint; et perutile foret, cuiusque speciei historiam hoc respectu observari, ut e multarum comparatione ad universalia progredi possimus et extricare, quae coeli solique potissimum mutationes paucis, quae multis speciebus aut prosint, aut exitium parent, quae artis molimina his, illisve obveniant. Sed nimirum cant et circumspicienter de causis exponi debet, quum et simillimarum specierum analogia saepe nobis illudat. Sic enim vere anni I 795 ., quo non magna insectorum noxiarum turbae exstabant, larvae noctuae oxyacanthae et bimaculosae solito frequentiores erant, etsi aliarum similium, verbi causa, noctuae Paranymphae, promissae, Auguris raritatem 
conquerebamur. Diuturna tamen et accurata omnium inspe: ctione specicium historia ita extricari deberet, ut, quae earum mutationum causa sit, inveniatur; sed antequam de causis loquamur, primum explicandum erit, quid mutationum contegerit; qua in re pauca huc usque facta esse dolemus, multa aliis, posteris, nobisque relinquimus. Tot enim adsunt, quae in historia insectorum facta esse accipiamus, quae e causis luculentis derivare "non possimus. Sic omnibus, Lipsiae ex annorum plurium serie insecta inquirentibus, testibus, multae species quondam frequentiores, nunc rariores sunt; inprimis ex his decem annis. Sic ante hoc tempus magnae turbae papilionis Iridis, populi, noctuae fraxini, larvarum bombycis processioneae, purpureae aliorumque exstabant, quorum iisdem locis hoc tempore vix unum aut alterum exemplum inveniatur. Iam si hosce casus silvarum rarefactioni, locorum aliorum eversioni et ex parte quoque animalculorum sedulae, per entomophilos susceptae, investigationi, illo tempore avidissimae, tribuamus, ea omnia sufficerent forsitan ${ }^{3}$ ad illud explicandum, quod aliquae species evanuerint; sed unde tot specierum caritàs originem traxerit; ex istis satis vixperspicio; ea certe, quae mox allegaverim, aton satis efficacia esse reor. Neque hisce et similibus observandis solis entomologis; sed maxime quoque oeconomis prodessemus, qui, dum quibus 
32

insecta sibi odiosissima possint pellere, didicerint, ex is unum vel alterum inveniant, quo, naturam imitati, inimicis libe. rentur; sed nimirum cum grano salis, hostiumque notitia fulti, ut ne forte pupas monachae aquarum artificiali immissione, suium inductione tollere constituant, aut chrysalides piniperdae in cortice quaerant.

Restat una res, quae insectorum propagationem impediat aut promoveat, migrationes puto, quas, etsi nulla a me unquam conspecta sit, e fide tamen tot tamque celebrium hominum, multum valere largior. Sed currendo istae fieri non faeile possunt, nisi forte larvas bombycis processioneae ex una in'alteram arborem repsisse, nigrare dicere velimus, quod ad unitiersae cuiusłam regionis casus referri nequit; sed migrationis voce hoc designandum est, si e loco remotiore subito magna animalculorum copia simul ad alterum se contulit. Sed hoc non potest fieri niși per insecta apte volitantia, quac itinera moliantur, aut valde levia, quae ventu impetuosissimo abripiantur, quod posterius ne notioni quidem exhibitae respondet, quum et raro per ventos insecta unius specici multa simul abripi sciamus, et ventorum, rapinăs non magis, quam aquae transportatianęs; - migrationum, activi modi vocabulo ornare audeamus. Nonnunquam vero etiam migrare insecta crediderunt, subito eodem tempore in, con- 
spectum prodeuntia, licet eodem loco natä et educta, huc usque tantum abscondita et imperfecta. . Et est omnino res; animadversu singularis, sàepe insectorum perfectorum cuiusdam speciei, ingentem molem uno die excurrere, 'quae eadem diverso tempore metamorphoseos initia subiissent, et facillime hoc patet, in erucis arte nutritis et oculorum obtutui sub. iectis. Srepe insecta in chrysalidis carcerem diversis diebus condita, ex improviso uno die omnia perfecta et matura aufligiunt. Eius vero phaenomeni causam ervere constanter repudiarem; autumo tamen, insecta in chrysalide manere etiam posteaquam plene formatae sint et excreverint, usque dum aeris sibi optime convenientis sensu excitati, arbitrio suo carceres rumpant; quo enim alio pacto fieret, ut multa simul eadem hora progrediantur; nec ea solum, quae per tantna temporis intervallum inertia chastris teneantur, ut unius aut aliẹnorum dierum spatium, tempori, quod ad ipsa evolvenda necessarium sit, paitem notabilem subtrahat aut addat: sed ea quoque quae tanium per paucas septimanas et vel adeo per octo dies solos quiescant. Iam quum, quae uno die egrediantur, diverso tempore in puparum formam transierint, eequitui", ea diverso temporis spatio egere; ad corporis partes satis evolvendas; sed quum omnia perfecta compareant: nihil aliud mihi fingere possum, nisi alia, eaque prius in 
nidulum ingressa et ocyus efformata, tamdiu sponte remansisse usquedum coeli temperiem sibi bene convenires praevidissent. Non dicat aliquis, esse hoc causuí fortuito tribuenduu, siquidem sexcenties observavi; nec onmibus perpensis interdicere mihi potui, quominus tempestatis causam in ius yocarem. Nempe saepissime id fieri perspexi tempestate humidiuscula et calidiore, frigora sicca aut aestus vehementes insequente; idque commodo simul animalium summo est; tum enim, quae teria reconduntur hanc molliorem facilius pertundunt, quae filis sese ubique cingunt, per ea aptius membra protrudunt, omnia vera melius artus evolvunt, quorum in vasis humorum ingens copia circumferenda, ridigitate vasorum, per exsiccationem aut frigoris iniuriam orta, stagnat, et ita omne incrementum impeditur: aere miti leniterque humido placide se expandunt alarunx rudinenta.

Sed satis superque perspectum habeo et expertum, diffcilis diuturnique laboris esse eiusmodi observationes: quare et propositi laboris molesti, et non ex asse conditi, veniam non omnem defuturam sperare liceat. Saepe hoc mente vol: vebam, omnium classium insecta ita studiose examinare, eorumque vitae victusque rationem tam indefesso studio, quo in muscorum historia, mire impedita, Hedwiginm, heu nobis ereptum, enitentem admirabamur, omni modo persequi, ut non 
ludibrio otia trivisse sed parvorum studio historiam naturalem et inprimis physiologiam utiliter ditasse, usuique communi aliquid saltim praestsitisse, de me, profiteri possim. In iisque ita versandum arbitrabar, ut primum specicrum omnium lo. cum et nomina.systematica cognoscerem, quo magis; quaenam animalcula observarem, eluceret, deinde quae cuique speciei singularia et propria sint; tum, quae multa inter se communia habeant; tandem quae generalia exinde colligi queant; enuclearem. Eius quidem propositi iam prima rudimenta exhibeo et de agri natura primum egi. Proximum est, ut sle systematica insectorum notitia, quae sciam, afferam; cuius occasio proxime edendi facultatem largietul. - Et omnino laetor in elaboranda topographia, olim plena et absoluta proponenda, non uni soli dimicandum esse, sed fautorem et doctoren me nactum esse V.Cl. LvDWrgrvm, qui et sua rerum naturalium scientia eximia, et studio indefesso et sibi amicorum concursu, naturalem Saxoniae conditionem scrutetur et notam reddat. Quorum quidem laborum primitias, in libro*), nuperrime societatis Linneanae nomine edito et catalogum insectorum Saxoniae in-

*) Erste Aufzählung der bis jetzt in Sachsen entdekten Inseliter. Im Namen der Linnéischen Societät herausgegeben von D. Christian Friedrich Ludwig, Prof. zu Leipzig. 'Leipziğ, in 'Commission bey Rabenhorst. 1799 . 
digenorum sistente, naturalis historiae cultares áccepere; iuxta quem catalogum meum tolerari posse propterea sperare ausus sum, quia iste meis parten Saxoniae singularum complectitur, et ad topographiae delineationem requiri videtur; maxime vero quia $\mathrm{Y}$. Cl. Ludwigius, fator summe colendus, ut tentaminis huius, quantum possem, publice proponerem, auctor suasorque fuit. Iamque scripsi eorum quae iusto nomire indita et a me ipso in agro nostro inventa habebam, recensum; quo. rum quidem copia augeri facile potuisset, si Clar. Ludwigii, Eberhardi, defuncti e paucis abhinc septimanis, et Zschachii coleopterorum ditiora receptacula fufficiente otio perlustrare, ab iisque viris, quantum vellem, discére potuissem; nam deficiente temporis otiique libertate, dierum hibenutium obscuritate, impeditus, quae illi possident, vix fugitivo oculo percurrere potui, nedum studiose examinare, compararo et super iis interrogare; etsi illorum virorum facilitatem et amicitiam erga me, non demeritam, collaudandum habeo. Neque praetermittere hic occasionem possum Dominum Zschachium publice iterum precandi, velit ea, quae de insectis Lipsiensibus collegerit et erudite conscripserit, quippe rerum novarum, observationum accuratarum, notarum gravissimarum plena, publici inris fiacere. Quod si fecisset, mea conamina aut supressissem, aut mire adiuta, meliora proposuissem. 



\section{.}




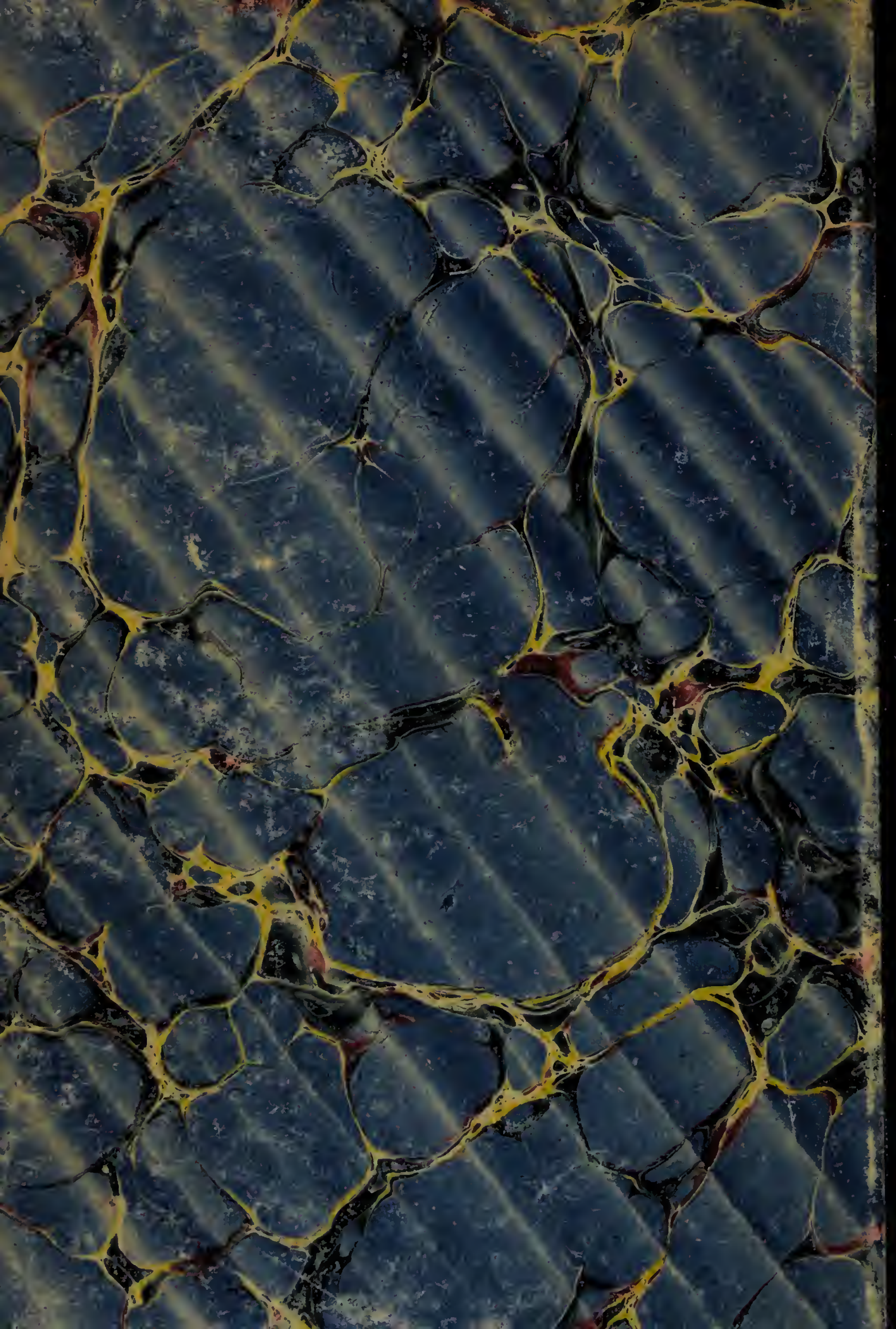

